This item was submitted to Loughborough's Institutional Repository (https://dspace.lboro.ac.uk/) by the author and is made available under the following Creative Commons Licence conditions.

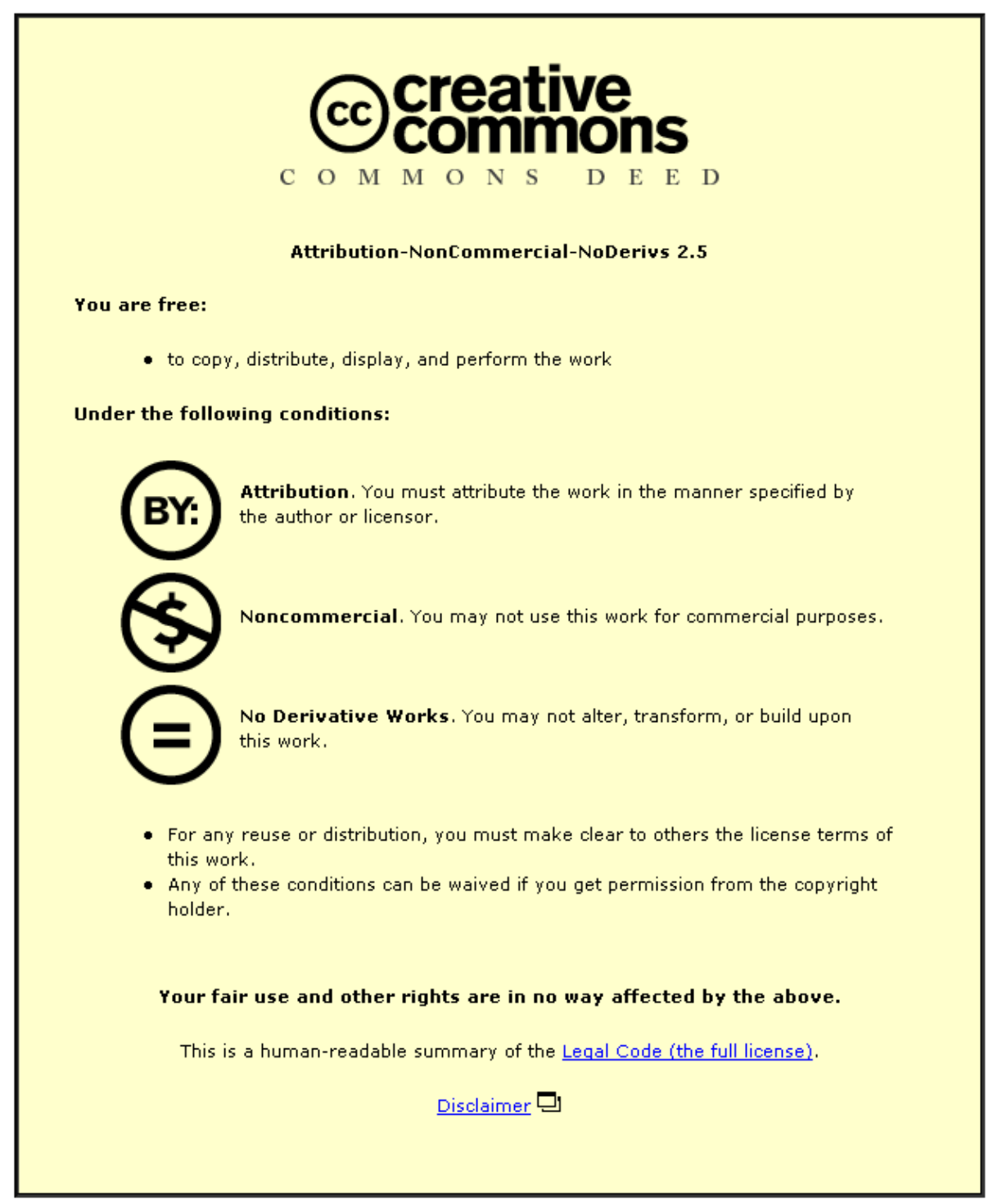

For the full text of this licence, please go to: http://creativecommons.org/licenses/by-nc-nd/2.5/ 


\title{
Energy downconversion between classical electromagnetic fields via a quantum mechanical SQUID ring
}

\author{
M. J. Everitt, ${ }^{1,2, *}$ T. D. Clark,,${ }^{1, \dagger}$ P. B. Stiffell, ${ }^{1}$ J. F. Ralph, ${ }^{3}$ and C. J. Harland ${ }^{1}$ \\ ${ }^{1}$ Centre for Physical Electronics and Quantum Technology, School of Science and Technology, University of Sussex, \\ Falmer, Brighton, BN1 9QT, United Kingdom \\ ${ }^{2}$ The British University in Egypt, 4 Khalid Ibn Walid Street, Sheraton Heliopolis Area, Cairo, Egypt \\ ${ }^{3}$ Department of Electrical and Electronic Engineering, Liverpool University, Brownlow Hill, Liverpool, L69 3GJ, United Kingdom
}

(Received 17 May 2005; published 16 September 2005)

\begin{abstract}
We consider the interaction of a quantum mechanical SQUID ring with a classical resonator (a parallel $L C$ tank circuit). In our model we assume that the evolution of the ring maintains its quantum mechanical nature, even though the circuit to which it is coupled is treated classically. We show that when the SQUID ring is driven by a classical monochromatic microwave source, energy can be transferred between this input and the tank circuit, even when the frequency ratio between them is very large. Essentially, these calculations deal with the coupling between a single macroscopic quantum object (the SQUID ring) and a classical circuit measurement device where due account is taken of the nonperturbative behavior of the ring and the concomitant nonlinear interaction of the ring with this device.
\end{abstract}

DOI: 10.1103/PhysRevB.72.094509 PACS number(s): 74.50.+r, 85.25.Dq, 03.65.Ud, 03.65.Yz

\section{INTRODUCTION}

With the now very serious interest being taken in the possibilities of creating quantum technologies such as quantum information processing and quantum computing,,${ }^{1-3}$ much attention is being focused on the application of Josephson effect devices, particularly the SQUID ring. As has been demonstrated recently, with the appropriate ring circuit parameters and operating temperature these devices can display manifestly macroscopic quantum behavior such as macroscopically distinct superposition of states. ${ }^{4-11}$ In any considerations of quantum technologies the role of the environment, as coupled to the quantum object, is featured very strongly. ${ }^{12-14}$ With regard to using superconducting systems in quantum technologies, it has been shown that Josephson weak link circuits, and in particular SQUID rings in the quantum regime, are highly nonperturbative in nature and can generate very strong nonlinear interactions with classical circuit environments. ${ }^{15-18}$ In this paper we provided a demonstration that this nonperturbative (nonlinear) behavior is crucial to the understanding of the interaction of SQUID rings with circuit environments. In this work we first consider the adiabatic (ground state) interaction of a quantum regime SQUID ring inductively coupled to a classical parallel resonance $L C$ (tank) circuit. This circuit system is shown schematically in Fig. 1(a). We then continue to consider energy transfer between a classical monochromatic microwave source (the input) and this classical electromagnetic (EM) field mode (the output) via the quantum mechanical SQUID ring [Fig. 1(b)]. Here, as in other work, ${ }^{19}$ we have modelled this output mode as a parallel $L C$ equivalent circuit. However, in this paper we have treated this circuit environment as classical since we wish to explore a region of the system parameter space, involving large input-output frequency ratios, which is of current experimental significance. When treated fully quantum mechanically, such simulations are beyond the reach of the computing power available to us. As the essential result of this paper, we show that the nonperturbative (nonlinear) interaction generated by the SQUID ring is made manifest through energy conversion between the input and output, even if these differ greatly in frequency. In part this has been inspired by past experimental results where we have demonstrated ${ }^{20}$ that very high ratio frequency downconversion can occur between a microwave source and a radio frequency $(\approx 20 \mathrm{MHz})$ tank circuit via the intermediary of a very small capacitance SQUID ring. ${ }^{20}$ In these previous results we recorded a frequency downconversion ratio of 500:1. In recent, as yet to be presented data, this has been improved upon by refinements in electronic technique to

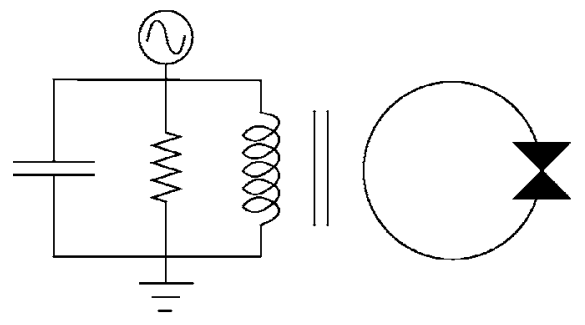

(a)

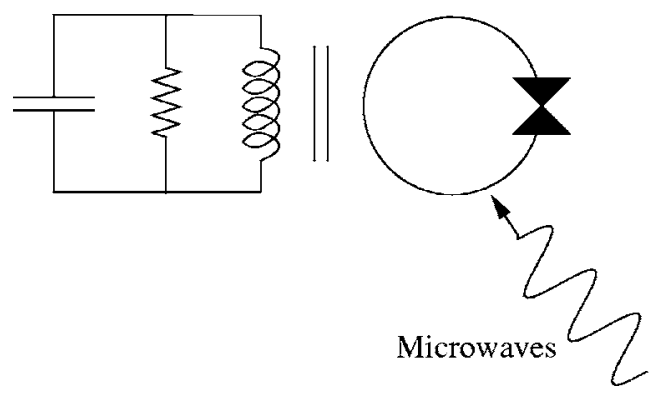

(b)

FIG. 1. (a) Schematic of a (quantum regime) SQUID ring inductively coupled to a driven tank circuit (two mode system), (b) schematic of a three mode system, classical input microwave excitation source, SQUID ring, and an inductively coupled tank circuit. 
yield a ratio as high as 6500:1 between a a few $\mathrm{GHz}$ microwave input field and a sub-MHz tank circuit. We know of no classical nonlinear circuit device that could generate such large ratios ${ }^{21}$ and, given the very small capacitance (niobium point contact) SQUID rings we were using in our experiments, it was clearly of interest to see whether a quantum regime SQUID ring could lead to this massive frequency downconversion. In a recent presentation ${ }^{22}$ we considered this problem in the context of the persistent current qubit circuit model introduced by Orlando et al. ${ }^{23}$ where this quantum element acted as the medium for coupling two classical field environments together. Specifically, the qubit was driven by a classical electromagnetic (EM) field (the input) which was used to pump the qubit into an excited state. To deal with energy conversion to a dissipative output EM mode, a quantum jumps approach was adopted to model the decay of the excited qubit with the energy dumped into this mode. Using this approach we were able to demonstrate small ratio (500 MHz to $300 \mathrm{MHz}$ ) frequency downconversion from input to output through the intermediary of the quantum qubit loop. In this work, with a single weak link SQUID ring as the quantum intermediary, we adopt a less complicated model, where the SQUID ring simply follows Schrödinger evolution without the introduction of quantum jumps. We then show, to the limits of the computational power available to us, that a two orders of magnitude frequency downconversion (from microwave to radio frequencies) is possible. In doing so we also demonstrate that the interaction between a quantum mechanical SQUID ring and its classical circuit environment is in no way trivial and requires a detailed understanding of the nonperturbative properties of SQUID rings. We emphasize that with this result established, and without any other obvious physical constraints, it seems reasonable to assume from a theoretical viewpoint that even higher ratio frequency downconversion could be realized if the necessary computational power were available. At present, apart from essentially fixed frequency masers, sources of quantum mechanical EM fields at microwave frequencies are not readily available. Thus, given the nonperturbative properties of SQUID rings in the quantum regime, it also seems reasonable to consider how such devices could manifest their quantum mechanical nature through the interaction with classical EM fields and field oscillator modes.

In the well known lumped component model of a quantum mechanical SQUID ring (in this work one Josephson weak link device, of effective capacitance $C_{s}$, enclosed by a thick superconducting ring of inductance $\Lambda_{s}$, with a classical magnetic flux of $\Phi_{x}$ applied to the ring) the Hamiltonian for the ring is given by ${ }^{24,25}$

$$
H_{s}=\frac{\hat{Q}_{s}^{2}}{2 C_{s}}+\frac{\left(\hat{\Phi}_{s}-\Phi_{x}\right)^{2}}{2 \Lambda_{s}}-\hbar \nu \cos \left(\frac{2 \pi \hat{\Phi}_{s}}{\Phi_{0}}\right),
$$

where, with a circumflex denoting operators, $\hat{\Phi}_{s}$ (the magnetic flux threading the ring of the SQUID device) and $\hat{Q}_{s}$ (the Maxwell electric displacement flux between the electrodes of the weak link) are canonically conjugate quantum

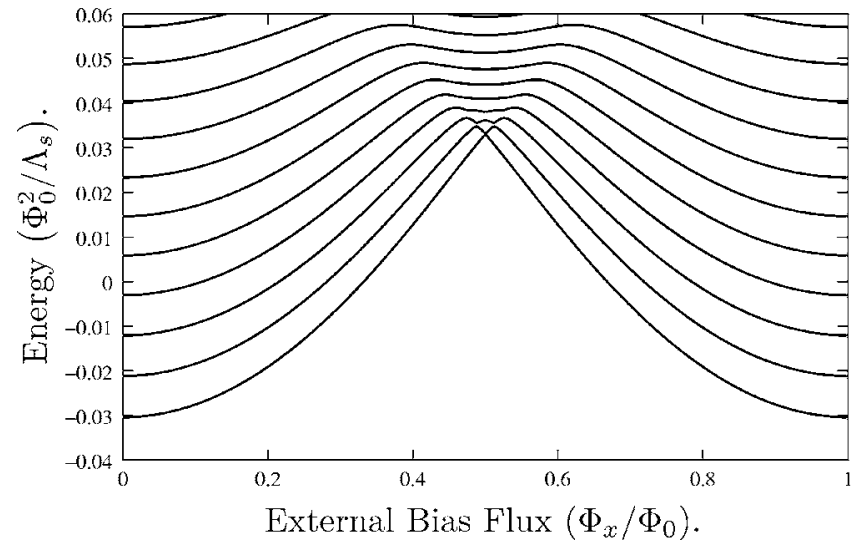

FIG. 2. Plot of the eigenenergies of the SQUID ring Hamiltonian, found by solving the TISE, as a function of external applied flux with $\hbar \omega_{s}=0.006 \Phi_{0}^{2} / \Lambda_{s}$ and $\hbar \nu=0.035 \Phi_{0}^{2} / \Lambda_{s}$.

variables such that $\left[\hat{\Phi}_{s}, \hat{Q}_{s}\right]=i \hbar$ with $\hat{Q}_{s} \rightarrow-i \hbar \partial / \partial \Phi_{s}$ and $\Delta \hat{\Phi}_{s} \Delta \hat{Q}_{s} \geqslant \hbar / 2$. Here, the third term on the right-hand side of (1) is due to the Josephson phase coherent coupling energy, with matrix element $\hbar \nu / 2$, arising from the weak link critical current $I_{c}=2 e \nu$, with periodicity set by the superconducting flux quantum $\Phi_{0}=h / 2 e$. We assume that the ambient temperature $(T)$ of the SQUID is such that $\hbar \omega_{s} \gg k_{B} T$ for a characteristic ring oscillator frequency of $\omega_{s} / 2 \pi\left(=1 / 2 \pi \sqrt{\Lambda_{s} C_{s}}\right)$. Operating in this quantum regime the time-independent Schrödinger equation (TISE) for the SQUID ring then reads

$$
\left[\frac{\hat{Q}_{s}^{2}}{2 C}+\frac{\left(\hat{\Phi}_{s}-\Phi_{x}\right)^{2}}{2 \Lambda}-\hbar \nu \cos \left(\frac{2 \pi \hat{\Phi}_{s}}{\Phi_{0}}\right)\right]\left|\psi_{\kappa}\right\rangle=E_{\kappa}\left|\psi_{\kappa}\right\rangle
$$

for ring eigenfunctions $\left|\psi_{\kappa}\right\rangle$ ( $\kappa=0$ the ground state, $\kappa=1$ the first excited state, etc.) and ring eigenenergies $E_{\kappa}$ (as shown in Fig. 2), these eigenenergies being $\Phi_{0}$ periodic in the external magnetic flux applied to the ring.

\section{DYNAMICS OF A COUPLED QUANTUM SQUID RING-CLASSICAL RESONATOR SYSTEM}

\section{A. Adiabatic regime}

In the time-independent (adiabatic) case the experimentally accessible state is the ground state $(\kappa=0)$ for which the expectation value of the screening supercurrent flowing around the ring is $\left\langle\hat{I}_{s}\left(\Phi_{x}\right)\right\rangle=-\left\langle\partial \hat{H} / \partial \Phi_{x}\right\rangle=\left\langle\left(\hat{\Phi}_{s}-\Phi_{x}\right) / \Lambda_{s}\right\rangle^{20,24}$ For relatively large values of $\hbar \nu$ (and hence $I_{c}$ ) this ground state screening current takes the form of a rounded sawtooth centered on $\Phi_{x}=\Phi_{0} / 2$ (modulo $n \Phi_{0}, n$ integer). Thus, the ring screening current is clearly a highly nonlinear function of $\Phi_{x}$, as is its magnetic susceptibility $\chi_{s}=\partial\left\langle\hat{I}_{s}\left(\Phi_{x}\right)\right\rangle / \partial \Phi_{x}$. At $\Phi_{x}=(n+1 / 2) \Phi_{0}, n$ integer, the ring is in a quantum superposition, with equal coefficients, of clockwise and anticlockwise screening currents (equivalently flux states). A rounded sawtooth jump in the screening current, centered at halfinteger bias flux, correspondingly generates a narrow positive spike in $\chi_{s}\left(\Phi_{x}\right)$ at this flux value. At integer bias flux $\left(\Phi_{x}=n \Phi_{0}\right)$ the susceptibility is a minimum, increasing mono- 
tonically to a maximum at $\Phi_{x}=(n+1 / 2) \Phi_{0}$. As we have shown, in this simple ground state model of a quantum regime SQUID ring the evolution of its quantum state can be monitored through its effect on the classical dynamics of a measurement circuit coupled to it. ${ }^{15,20,24}$ Typically this takes the form of a low frequency, parallel $L C$ tank circuit inductively coupled to the SQUID ring and excited at, or very close to, its resonant frequency. The resonant frequency of this tank circuit $\left(\omega_{t} / 2 \pi\right)$ is usually extremely low compared with the natural oscillator frequency of the SQUID ring (with the implicit assumption that $\left.\hbar \omega_{t} \ll k_{B} T\right)$. This configuration is very well known and forms the basis for one type of ultrasensitive magnetic flux detector-the ac or radio frequency biased SQUID magnetometer. ${ }^{25}$ With this tank circuit playing the role of the classical measurement system for the SQUID ring, a changing $\chi_{s}\left(\Phi_{x}\right)$ leads, in the ground state of the ring, to downward shifts [due to the positive $\chi_{s}\left(\Phi_{x}\right)$ ] of the resonant frequency of the tank circuit. For this ground state, therefore, following the change in the frequency (and amplitude) of the tank circuit resonance allows us to extract some information concerning the evolution of the quantum state of the ring (i.e., the coefficients in its screening current superposition) as a function of $\Phi_{x}$.

\section{B. The Born-Oppenheimer approximation}

Given that the oscillator frequencies of both the SQUID ring and the (classical) tank circuit resonator are functions of their circuit capacitances, it is, in this limit, often convenient to introduce an approach well known in atomic physics. These capacitances can be considered as measures of the effective mass of each circuit (SQUID ring and tank circuit resonator). Crucially, for quantum regime SQUID rings these capacitances (effective masses) will be markedly different in magnitude, e.g., for the ring typically $\approx \mathrm{a}$ few $\times 10^{-15} \mathrm{~F}$ and for the tank circuit $\approx$ a few $\times 10^{-11} \mathrm{~F}$, or more. This low mass-high mass (here, low capacitance-high capacitance) situation was dealt with many years ago by Born and Oppenheimer for nuclear-electron motion. Adopting this approach we compute from solutions of (2) the expectation value of the screening supercurrent in the SQUID ring (fast) and substitute this as a feedback term into the classical equation of motion for the tank circuit (slow). Provided that the resonant frequency of the tank circuit is low enough, so that to an extremely good approximation the quantum SQUID ring remains adiabatically in its ground state, the equation of motion for the coupled ring-tank circuit system then reads ${ }^{15,24}$

$$
C_{t} \frac{\partial^{2} \Phi_{t}}{\partial t^{2}}+\frac{1}{R_{t}} \frac{\partial \Phi_{t}}{\partial t}+\frac{1}{L_{t}} \Phi_{t}=I_{\mathrm{in}}(t)+\mu\left\langle\psi\left|\hat{I}_{s}\right| \psi\right\rangle,
$$

where $L_{t}$ and $C_{t}$ are, respectively, the tank circuit inductance and capacitance, $\Phi_{t}$ is the magnetic flux in the tank circuit inductor, and $R_{t}$ is the resistance of the tank circuit on resonance. In this Born-Oppenheimer approximation we assume that the ring remains in one of its instantaneous energy eigenstates, i.e., solutions of (2); in reality this means the lowest energy state (the adiabatic limit). As we have demonstrated in the literature, ${ }^{26}$ this approximation holds very well if the frequency of the drive is very small indeed compared

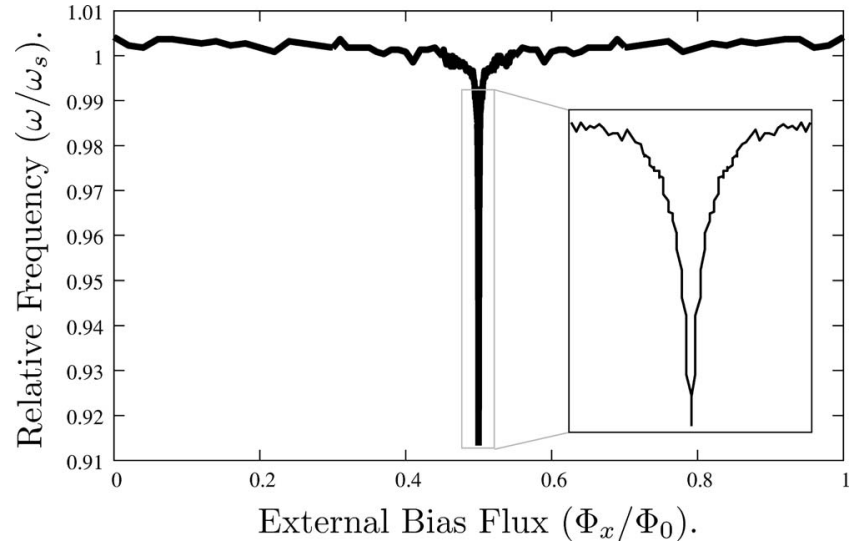

FIG. 3. Frequency shift of a $130 \mathrm{MHz}$ tank circuit $\left(L_{t c}=3\right.$ $\times 10^{-8} \mathrm{H}, C_{t c}=5 \times 10^{-11} \mathrm{~F}$ ), coupled to the SQUID ring of Fig. 2, as a function of external applied flux for the ring in its ground state only [Fig. 2(a)] calculated using the Born-Oppenheimer (adiabatic) approach.

with any separations, in frequency, between the SQUID ring energy levels. This certainly appears to be the case for radio frequency (rf) circuits, typically used to probe single weak link SQUID rings, as well as in their application in SQUID magnetometry. ${ }^{27,28}$ We note that since the SQUID ring is macroscopic in nature (as, of course, is the tank circuit) there exists a significant back reaction between these two circuits, as evidenced by the $\mu\left\langle\psi\left|\hat{I}_{s}\right| \psi\right\rangle$ term in (3). In practice this means that the SQUID ring (through the cosine in its Hamiltonian) generates a nonlinear dynamic in the classical circuit environment to which it is coupled, in this case a tank circuit. For small tank circuit drive amplitudes this manifests itself as a frequency shift in the power spectrum of the tank circuit as shown in Fig. 3.

\section{TIME-DEPENDENT SCHRöDINGER EQUATION DESCRIPTION-EXCHANGE REGIONS}

Following the time-independent Schrödinger description of a SQUID ring (2), the time-dependent Schrödinger equation (TDSE) for the ring takes the form

$$
\left[\frac{\hat{Q}_{s}^{2}}{2 C_{s}}+\frac{\left(\hat{\Phi}_{s}-\Phi_{x}\right)^{2}}{2 \Lambda_{s}}-\hbar \nu \cos \left(\frac{2 \pi \hat{\Phi}_{s}}{\Phi_{0}}\right)\right]|\psi\rangle=i \hbar \frac{\partial|\psi\rangle}{\partial t},
$$

where $\Phi_{x}=\Phi_{x}^{\text {stat }}+\Phi(t)$ is now comprised of a time- dependent magnetic flux $\Phi(t)$ together with a static component $\Phi_{x}^{\text {stat }}$. The intrinsically extremely nonperturbative quantum nature of the SQUID ring would indicate that the application of EM fields low in frequency compared with the frequency difference $(\times h)$ between adjacent energy levels in the ring should still induce transitions between its quantum levels. ${ }^{29}$ Thus for level differences of a few hundred $\mathrm{GHz}$ (quite typical of quantum regime SQUID rings), we expect EM fields at microwave frequencies (a few $\mathrm{GHz}$ ) to induce transitions, provided the field amplitude is high enough. ${ }^{29}$ As our example in this work we consider a SQUID ring with circuit parameters commensurate with operation in 


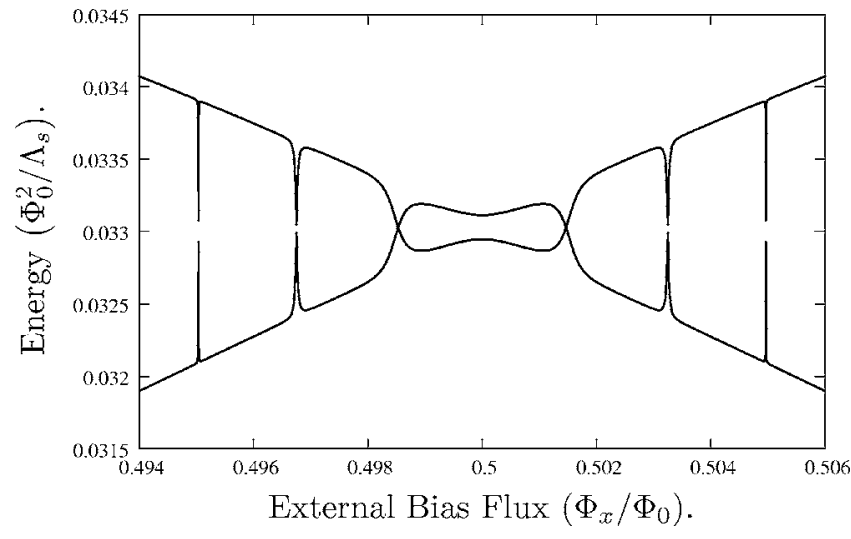

FIG. 4. Time averaged energy expectation values of the ring in the presence of a microwave source (input) at a frequency of $13 \mathrm{GHz}$ using the first two energy eigenstates as initial conditions.

the quantum regime. ${ }^{2,30}$ For this we choose $C_{s}=5 \times 10^{-15} \mathrm{~F}$, $\Lambda_{s}=3 \times 10^{-10} \mathrm{H}$, and $\hbar \nu=0.035 \Phi_{0}^{2} / \Lambda_{s}$, the latter yielding a weak link critical current $I_{c}(=2 e \nu)$ of $2 \mu \mathrm{A}$. Furthermore, for an oxide insulator, tunnel junction weak link (oxide thickness $\approx 1 \mathrm{~nm}$, oxide dielectric constant $\approx 10$ ) in the SQUID ring, this capacitance corresponds to junction dimensions $0.25 \mu \mathrm{m}^{2}$, well within the capabilities of modern microfabrication technology. In turn, these dimensions imply a maximum supercurrent density in the weak link of around $4 \mathrm{kA} \mathrm{cm}^{-2}$, a perfectly reasonable value for current experiments on quantum regime SQUID rings. The SQUID ring circuit parameters $C_{s}=5 \times 10^{-15} \mathrm{~F}$ and $\Lambda_{s}=3 \times 10^{-10} \mathrm{H}$ lead to a ring oscillator frequency $\omega_{s} / 2 \pi=130 \mathrm{GHz}$. If we assume that the (planar) SQUID ring is fabricated in niobium, which is often the case, then this ring oscillator frequency is small compared with the superconducting energy gap in this material $(\approx 1000 \mathrm{GHz})$ at low reduced temperatures $T / T_{c}$, where $T_{c}$ is the superconducting critical temperature $(9.2 \mathrm{~K}$ for nio bium) for an operating (ambient) temperature in experiment typically around $40 \mathrm{mK}$.

Using the ring circuit parameters given above we showed in Fig. 2 the lowest few energy eigenvalues of the SQUID ring Hamiltonian, found by solving the TISE (4), as a function of the static applied magnetic flux $\Phi_{x}^{\text {stat }}$. Although such solutions of the TISE may be adequate for modelling this system if the EM fields frequencies are extremely small compared with frequencies $(\times h)$ separating the ring energy levels (i.e., in the Born-Oppenheimer approximation, above), in general we must solve the TDSE where time-dependent fields are involved. This is clearly demonstrated in Fig. 4. Here, using the ring parameters of Fig. 2, we display the time averaged energy expectation values of the TDSE (4) as a function of $\Phi_{x}^{\text {stat }}$, for an applied microwave field of frequency $13 \mathrm{GHz}$, amplitude $0.001 \Phi_{0}$ and using the first two eigenstates of the Hamiltonian as initial conditions. As can be seen, for this microwave frequency and amplitude there is significant energy exchange between these time averaged energies which takes place over a very narrow range of the bias flux $\Phi_{x}^{\text {stat }}$. We term these exchange regions, ${ }^{19,29}$ or equivalently, transition regions. We note that at the centers of these exchange regions the corresponding frequency differences between the energy eigenenergies in Fig. 2 are close to integer multiples of the applied microwave frequency. At sufficiently high EM frequencies and amplitudes several, or many, exchange regions are generated with an energy spacing between adjacent regions very close to $\hbar \omega_{\mathrm{mw}}$, where $\omega_{\mathrm{mw}}$ is the (angular) frequency of the EM field. As we have shown in a previous presentation, ${ }^{19}$ it is in these exchange regions that the interaction between a SQUID ring and one or more EM fields, is significant. For example, when a field mode, or modes, is treated quantum mechanically, entanglement is generated between the ring and the mode(s), reaching (as with the strength of the interaction) a maximum in the center of an exchange region. Given the result shown in Fig. 4 , where it is clear that energy is being exchanged between the SQUID ring and the (classical) EM field, we now consider whether a similar exchange can take place between this input field and a classical output field mode, coupled together through the ring.

We now investigate a scenario, accessible at the current level of experimental practice, ${ }^{20}$ of a classical field input and a classical field mode output, differing widely in frequency. We demonstrate that energy can be exchanged via a highly nonperturbative, quantum mechanical SQUID ring. In particular, and in line with this experimental background, ${ }^{20}$ we consider the interaction and energy transfer via the SQUID ring between a microwave input, acting purely as a source, and a lower frequency, resonant circuit output. This models the SQUID ring-tank circuit (ac-biased) magnetometer configuration $^{27}$ in a nonadiabatic EM (microwave) field. In this paper, we assume that there is a macroscopically significant back reaction between the tank circuit and the SQUID ring. ${ }^{15,31}$ However, for simplicity, we assume that there is no direct coupling between the EM input and output fields and note that this is easy to realize experimentally.

In order to deal in a general manner with this coupled ring-tank circuit system we can no longer assume that the behavior of this system is adiabatic in nature. By implication, this means that the tank circuit resonant frequency is sufficiently large compared with the frequency separations between the SQUID ring levels. Hence, we can no longer invoke the Born-Oppenheimer approximation. The TDSE for the system then takes the form ${ }^{27}$

$$
\begin{aligned}
& {\left[\frac{\hat{Q}_{s}^{2}}{2 C_{s}}+\frac{\left[\hat{\Phi}_{s}-\left(\Phi_{x}^{\mathrm{stat}}+\Phi_{x}^{\mathrm{mw}}+\mu \Phi_{t}\right)\right]^{2}}{2 \Lambda_{s}}-\hbar \nu \cos \left(\frac{2 \pi \hat{\Phi}_{s}}{\Phi_{0}}\right)\right]} \\
& \quad \times|\psi(t)\rangle=i \hbar \frac{\partial|\psi(t)\rangle}{\partial t}
\end{aligned}
$$

where, as can be seen, the total external magnetic flux applied to the SQUID ring consists of static bias, microwave excitation, and back reaction tank circuit contributions, i.e., $\Phi_{x}=\Phi_{x}^{\text {stat }}+\Phi_{x}^{\mathrm{mw}}+\mu \Phi_{t}$. Again, as with the Born-Oppenheimer approximation (3), we assume that this back reaction is macroscopically significant.

Given that the SQUID ring is now allowed to follow a Schrödinger evolution and retain its time dependence (3) in this nonadiabatic regime, the equation of motion for the tank circuit now becomes 


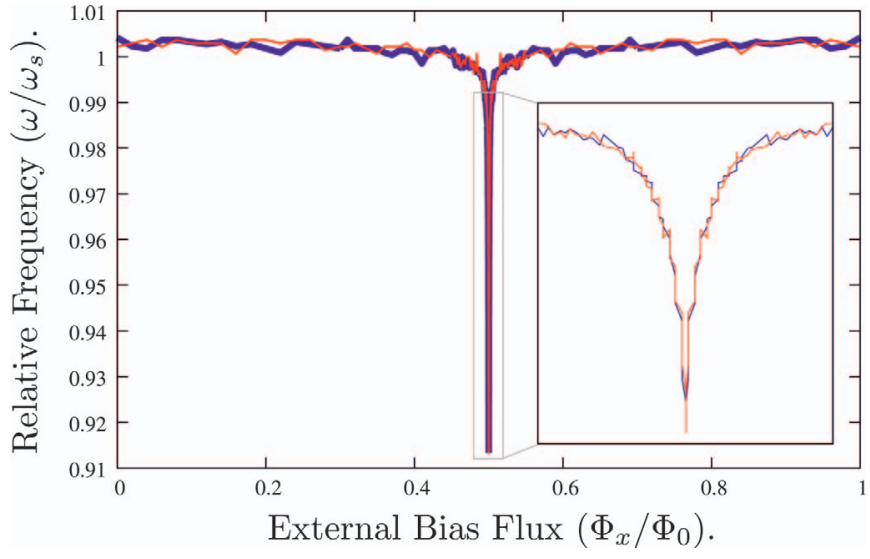

FIG. 5. (Color) Frequency shift of a $130 \mathrm{MHz}$ tank circuit $\left(L_{t c}\right.$ $=3 \times 10^{-8} \mathrm{H}, C_{t c}=5 \times 10^{-11} \mathrm{~F}$ ), coupled to the SQUID ring of Fig. 2 , as a function of external applied flux for the ring in its ground state only (Fig. 2) calculated using both the Born-Oppenheimer (in blue) and the nonadiabatic (in red) approaches.

$$
C_{t} \frac{\partial^{2} \Phi_{t}}{\partial t^{2}}+\frac{1}{R_{t}} \frac{\partial \Phi_{t}}{\partial t}+\frac{1}{L_{t}} \Phi_{t}=I_{\mathrm{in}}(t)+\mu\left\langle\psi(t)\left|\hat{I}_{s}(t)\right| \psi(t)\right\rangle,
$$

where

$$
I_{s}=-\frac{\partial \hat{H}_{s}}{\partial \Phi_{x}}=\frac{\hat{\Phi}_{s}-\Phi_{x}(t)}{\Lambda_{s}} .
$$

We solve the simultaneous coupled differential equations (5) and (6), where the frequency ratio between input microwave and output tank circuit modes is large $(\geqslant 100)$. In our opinion these results shed light on both the general problem of the description of the quantum-classical interface and, in particular, the interaction of nonlinear devices such as SQUID rings with their classical environments.

\section{RESULTS}

As a first check of the validity of this model we compare it with the established Born-Oppenheimer approximation for the limiting regime where there are no microwaves applied and the tank circuit is driven at a frequency $f_{t}$ so low that, to an extremely good approximation, the SQUID ring remains adiabatically in its ground state. This holds even though, through solutions of (2), a whole spectrum of ring eigenstates is available. In order to demonstrate the correspondence between these two models in the limit of low tank circuit drive frequency, we calculate the $\Phi_{x}^{\text {stat }}$-dependent frequency shifts $f\left(\Phi_{x}^{\text {stat }}\right)$ using the ring parameters of Fig. 2 and a tank circuit resonant frequency $130 \mathrm{MHz}$ and a $\mu=0.01$. In Fig. 5 (as we did in Fig. 3) we show the computed ring-tank circuit resonant frequency shift in this limit as a function of $\Phi_{x}^{\text {stat }}$ for both the Born-Oppenheimer approach (in blue) and our generalized model (in red). It is quite apparent that there is a very high degree of agreement between the two approaches in this limit. As our example of the frequency conversion process we consider a SQUID ring with circuit parameters used in the computed results of Figs. 2 and 4. For the SQUID ring parameters we have chosen the characteris-

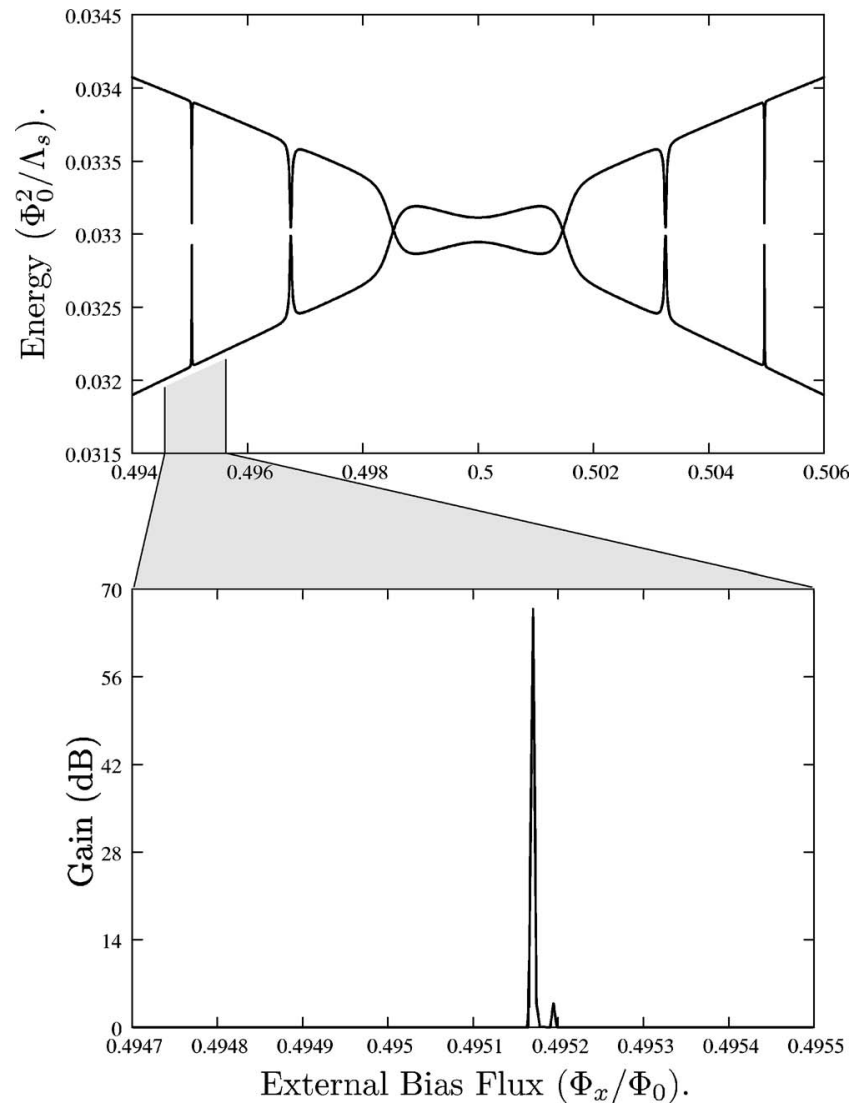

FIG. 6. (Top) Reproduction of Fig. 4 illustrating the region of bias flux used to compute (bottom) the power gain in the tank circuit (with respect to $\Phi_{x}^{\text {stat }}=0 \Phi_{0}$ ). Here, the microwave source frequency was $13 \mathrm{GHz}$ which converted into the output (tank circuit) oscillator mode of $130 \mathrm{MHz}$. As can be seen, the peak power at the center of the exchange region is $\approx 70 \mathrm{~dB}$ above the background from outside the exchange region.

tic ring oscillator frequency to be close to $130 \mathrm{GHz}$. Referring to the computed results of Fig. 4, we again choose the frequency for the microwave source to be $13 \mathrm{GHz}$, i.e., an order of magnitude lower than the ring characteristic frequency, and set the tank circuit resonant frequency another two orders of magnitude lower (i.e., $130 \mathrm{MHz}$ ). Within the limits of the computational power available to us this provides us with the opportunity to demonstrate extreme (1000:1), quantum SQUID ring mediated, energy downconversion.

With the results of Fig. 5 in support, and utilizing the time averaged energy expectation results of Fig. 4 as a guide, we then proceeded to compute the energy transfer, via the SQUID ring, from input field to an undriven output tank circuit at selected flux bias points close to and within an exchange region in Fig. 4. Our results are shown in Fig. 6 for the region of bias flux denoted using the time averaged energy expectation values at the top (which reproduces Fig. 4). As can be seen, outside the exchange region there is no evidence of energy conversion between the input microwave field and the output tank circuit. However, within the exchange region there is very significant conversion, reaching a maximum gain $(\approx 70 \mathrm{~dB}$ above background $)$ at its center. 
The results we have presented in this paper demonstrate that a input-output frequency downconversion of a factor of a couple of orders of magnitude can be explained through the simple model we have utilized. However, this does not constitute a theoretical upper limit, only a practical constraint arising from the level of computational power available to us. We also note that by symmetry, and due to the fact that the SQUID ring couples different frequencies together, frequency upconversion via this same mechanism should also be possible.

\section{CONCLUSIONS}

In this paper we have demonstrated that the interaction of a quantum mechanical SQUID ring with classical circuit environments is nontrivial. However, in the usual approach to the influence of a classical (and dissipative) circuit environment on the time evolution of a quantum mechanical SQUID ring, ${ }^{12}$ it is assumed that the environment can be modelled by a bath of linear harmonic oscillators linearly coupled to the ring. ${ }^{32}$ This need not be the case. The highly nonperturbative nature of the SQUID ring in the quantum regime (and other Josephson weak link based circuits) means that the ring- environment interaction can be very nonlinear and may lead to unexpected results. One of these is clearly the extreme frequency ratio conversion possible between classical fields via a quantum mechanical SQUID ring. Since the problem of environmental (dissipative, decohering) effects is so central to the successful implementation of quantum technologies, it is our opinion that for nonlinear devices such as SQUID rings the role of the environment must be reconsidered within this nonperturbative (nonlinear) context.

On a purely experimental level the theoretical results generated in this paper indicate that quantum SQUID rings can be used for very large frequency ratio downconversion between classical fields. This seems to be supported by experiment ${ }^{20}$ and may prove to be of considerable practical significance, especially with the current interest in classical THz communications technologies. ${ }^{33}$

\section{ACKNOWLEDGMENT}

The authors would like to thank the Engineering and Physical Sciences Research Council for supporting the work presented in this paper through its Quantum Circuits Network programme.
*Electronic address: m.j.everitt@physics.org

$\dagger$ Electronic address: t.d.clark@sussex.ac.uk

${ }^{1}$ H. Lo, S. Popescu, and T. P. Spiller, Introduction to Quantum Computation and Information (World Scientific, New Jersey, 1998).

${ }^{2}$ M. A. Nielsen and I. L. Chuang, Quantum Computation and Quantum Information (Cambridge University Press, Cambridge, 2000).

${ }^{3}$ The Physics of Quantum Information, edited by D. Bouwmeester, A. Ekert, and A. Zeilinger (Springer-Verlag, Berlin, 2000).

${ }^{4}$ C. H. van der Wal, C. J. ter Haar, F. K. Wilhelm, R. N. Schouten, C. J. P. M. Harmans, T. P. Orlando, S. Lloyd, and J. E. Mooij, Science 290, 773 (2000).

${ }^{5}$ J. R. Friedman, V. Patel, W. Chen, S. K. Tolpygo, and J. E. Lukens, Nature (London) 406, 43 (2000).

${ }^{6}$ Y. Nakamura, C. D. Chen, and J. S. Tsai, Phys. Rev. Lett. 79, 2328 (1997).

${ }^{7}$ Y. K. Nakamura, Y. A. Pashkin, and J. S. Tsai, Nature (London) 398, 786 (1999).

${ }^{8}$ J. M. Martinis, S. Nam, J. Aumentado, and C. Urbina, Phys. Rev. Lett. 89, 117901 (2002).

${ }^{9}$ P. Silvestrini, B. B. Ruggiero, C. Grananta, and E. Esposito, Phys. Lett. A 267, 45 (2000).

${ }^{10}$ I. Chiorescu, Y. Nakamura, C. Harmans, and J. E. Mooij, Science 299, 1869 (2003).

${ }^{11}$ T. P. Spiller, Fortschr. Phys. 48, 1075 (2000).

${ }^{12}$ U. Weiss, Quantum Dissipative Systems (World Scientific, Singapore, 1999).

${ }^{13}$ H. J. Carmichael, An Open Systems Approach to Quantum Optics, Lecture Notes in Physics, M18 (Springer, Berlin, 1993).

${ }^{14}$ A. J. Leggett, S. Chakravarty, A. T. Dorsey, M. P. A. Fisher, A. Garg, and W. Zwerger, Rev. Mod. Phys. 59, 1 (1987).
${ }^{15}$ T. D. Clark, J. F. Ralph, R. J. Prance, H. Prance, J. Diggins, and R. Whiteman, Phys. Rev. E 57, 4035 (1998).

${ }^{16}$ E. Ilichev, N. Oukhanski, A. Izmalkov, T. Wagner, M. Grajcar, H.-G. Meyer, A. Y. Smirnov, A. Maassen van den Brink, M. H. S. Amin, and A. M. Zagoskin, Phys. Rev. Lett. 91, 097906 (2003).

${ }^{17}$ A. Yu. Smirnov, Phys. Rev. B 67, 155104 (2003).

${ }^{18}$ A. Blais, A. M. van den Brink, and A. M. Zagoskin, Phys. Rev. Lett. 90, 127901 (2003).

${ }^{19}$ M. J. Everitt, T. D. Clark, P. Stiffell, A. Vourdas, J. F. Ralph, H. Prance, and R. J. Prance, Phys. Rev. B 64, 184517 (2001).

${ }^{20}$ R. Whiteman, T. D. Clark, R. J. Prance, H. Prance, V. Schollmann, J. F. Ralph, M. J. Everitt, and J. Diggins, J. Mod. Opt. 45, 1175 (1998).

${ }^{21}$ W. T. Silfvast, Laser Fundamentals (Cambridge University Press, Cambridge, 1996).

${ }^{22}$ J. F. Ralph, T. D. Clark, M. J. Everitt, H. Prance, P. B. Stiffell, and R. J. Prance, Phys. Lett. A 317, 199 (2003).

${ }^{23}$ T. P. Orlando, J. E. Mooij, L. Tian, C. H. van der Wal, L. Levitov, S. Lloyd, and J. J. Mazo, Phys. Rev. B 60, 15398 (1999).

${ }^{24}$ T. P. Spiller, T. D. Clark, R. J. Prance, and A. Widom, Progress in Low Temperature Physics (Elsevier Science, Amserdam, 1992), Vol. XIII.

${ }^{25}$ Y. Srivastava and A. Widom, Phys. Rep. 148, 1 (1987).

${ }^{26}$ R. Whitemen, V. Schollmann, M. J. Everitt, T. D. Clark, R. J. Prance, H. Prance, J. Diggins, G. Buckling, and J. F. Ralph, J. Phys.: Condens. Matter 10, 9951 (1998).

${ }^{27}$ O. V. Lounasmaa, Experimental Principles and Methods below $1 \mathrm{~K}$ (Academic, London, 1974), pp. 156-159.

${ }^{28}$ K. K. Likharev, Dynamics of Josephson Junctions and Circuits (Gordon and Breach, New York, 1986). 
${ }^{29}$ T. D. Clark, J. Diggins, J. F. Ralph, M. J. Everitt, R. J. Prance, H. Prance, R. Whiteman, A. Widom, and Y. N. Srivastava, Ann. Phys. 268, 1 (1998).

${ }^{30}$ M. J. Everitt, T. D. Clark, P. B. Stiffell, A. Vourdas, J. F. Ralph, R. J. Prance, and H. Prance, Phys. Rev. A 69, 043804 (2004).
${ }^{31}$ J. F. Ralph, T. D. Clark, M. J. Everitt, and P. Stiffell, Phys. Rev. B 64, 180504 (2001).

${ }^{32}$ A. O. Caldeira and A. J. Leggett, Ann. Phys. 149, 374 (1983).

${ }^{33}$ A. G. Davies, E. H. Linfield, and M. Pepper, Philos. Trans. R. Soc. London, Ser. A 362, 197 (2004). 